\title{
Hydrostatic pressure decreases the proton mobility in the hydrated $\mathrm{BaZr}_{0.9} \mathrm{Y}_{0.1} \mathrm{O}_{3}$ proton conductor
}

\author{
Qianli Chen, ${ }^{1,2}$ Artur Braun, ${ }^{1, a)}$ Alejandro Ovalle, ${ }^{1}$ Cristian-Daniel Savaniu, ${ }^{3}$ \\ Thomas Graule, ${ }^{1,4}$ and Nikolai Bagdassarov ${ }^{5}$ \\ ${ }^{1}$ Laboratory for High Performance Ceramics, Swiss Federal Laboratories for Materials Science \\ and Technology, Empa, CH-8600 Dübendorf, Switzerland \\ ${ }^{2}$ Department of Physics, Swiss Federal Institute of Technology, ETH Zürich, CH-8057 Zürich, Switzerland \\ ${ }^{3}$ School of Chemistry, University of St. Andrews, St Andrews, Fife, KY16 8DA Scotland, United Kingdom \\ ${ }^{4}$ Technische Universität Bergakademie Freiberg, D-09596 Freiberg, Germany \\ ${ }^{5}$ Institut for Geosciences, J. W. Goethe Universität Frankfurt am Main, D-60323 Frankfurt/Main, Germany
}

(Received 19 April 2010; accepted 22 June 2010; published online 27 July 2010)

\begin{abstract}
Impedance spectroscopy on the hydrated proton conductor $\mathrm{BaZr}_{0.9} \mathrm{Y}_{0.1} \mathrm{O}_{3}$ at high temperatures shows that the bulk proton conductivity activation energy $\mathrm{E}_{\mathrm{b}}$ scales with the strain parameter $\varepsilon$, as achieved by hydrostatic pressures up to $2 \mathrm{GPa}$, suggesting that large lattices favor proton diffusivity. At high temperature, $\mathrm{E}_{\mathrm{b}}$ increases upon pressure by $40 \%$. The grain boundary activation energy $\mathrm{Eg}$ is around twice as $E_{b}$, indicating higher proton mobility in grain boundaries as a result of pressure induced sintering. An expanded lattice with strain parameter $\varepsilon>1$ should have lower $\mathrm{E}_{\mathrm{b}}$, suggesting that thin films expansive tensile strain could have larger proton conductivity. (C) 2010 American Institute of Physics. [doi:10.1063/1.3464162]
\end{abstract}

Proton conducting ceramics have the potential for a wide range of applications, for example, solid electrolytes in electrochemical devices such as fuel cells, electrolyzers, and sensors. Compared to conventional solid electrolytes based on oxygen ion conductivity, materials with pronounced proton conductivity are attractive for lowering the operating temperature. Yttrium substituted barium zirconate, a perovskite with $\mathrm{ABO}_{3}$ structure, in which oxygen vacancies are created by $\mathrm{Y}$-substitution to the $\mathrm{Zr}$ at the $\mathrm{B}$-side, is an attractive candidate material. ${ }^{1}$ While the fundamental physical and chemical processes of the proton conductivity are not all well known, the current opinion is that in aqueous atmosphere, the vacancy can be filled by oxygen which dissociates from adsorbed water molecule, ${ }^{2}$ and the introduced protons are bound to the lattice oxygen in the octahedron $\mathrm{ZrO}_{6}{ }^{3}$ While literature has not been unambiguous about models and details, ${ }^{4,5}$ proton diffusion can be considered a combination of free diffusion and trapping and escape events. ${ }^{6,7}$ The first state represents free, delocalized diffusion of the protons jumping from one oxygen to another oxygen, forming $\mathrm{OH}^{-}$, and a very fast localized motion of the $\mathrm{OH}^{-}$as a reorientation; the second state represents protons trapped on energetically lowered sites near a dopant ion such as $\mathrm{Y}^{3+}$. The fast localized motion of $\mathrm{OH}^{-}$ions comes with an activation energy in the order of few millielectron volt at moderate temperature; ${ }^{6}$ whereas at elevated temperature, thermal activation may drive the proton to another oxygen site by overcoming an energy barrier in the range of a few hundred millielectron volt, ${ }^{7}$ and thus constituting charge mobility. Not only for improving device applications, but also from a fundamental point of view it is of high interest to understand the relationship of structure parameters and proton transport properties. The potential relations between proton conduction activation energy $\left(E_{a}\right)$ and structure parameters were pointed out as early as 1988 by Scherban, ${ }^{8}$ who found that

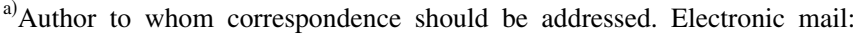
artur.braun@alumni.ethz.ch. Tel.: +41 44823 4850. FAX: +41 44823 4150.
}

the $E_{a}$ of $\mathrm{Yb}$-doped $\mathrm{SrCeO}_{3}$ and $\mathrm{BaCeO}_{3}$ decreases when their oxygen-oxygen distance increases. Our recent findings applying "chemical pressure" - achieved by different synthesis routes-suggest that $E_{a}$ for bulk proton transport increases with decreasing lattice spacing. ${ }^{, 10}$ In their work on $\mathrm{Ba}_{6-\mathrm{y}} \mathrm{Ca}_{\mathrm{y}} \mathrm{Nb}_{2} \mathrm{O}_{11}$, Ashok ${ }^{11}$ et al. found that an expansion of the unit cell caused by varying the concentration of the A-site element eases the carrier mobility. Wu et al. ${ }^{12,13}$ reported that heavily Y-doped $\mathrm{BaCO} 3$ exhibits significantly lower conductivity than those of lightly doped analogs, suggesting that facilitating proton transport based on B-site doping has limitations. While change in the A-site cation size has a direct effect on the lattice volume and also on the proton conductivity, one cannot generally rule out that the different chemical nature of the chosen substituent may have an additional specific influence. The direct method to prove the aformentioned hypothesis is to apply hydrostatic pressure $(p)$ - and temperature (T)-dependent analytical techniques to monitor the proton transport, as was done previously with impedance spectroscopy. ${ }^{14}$ To investigate intuitively the change in the lattice volume of the proton conductor, it is technically more challenging to expand the lattice volume. In this work, $\mathrm{BaZr}_{0.9} \mathrm{Y}_{0.1} \mathrm{O}_{2.95}$ (BZY10) was prepared and the structure determined by $\mathrm{X}$-ray diffraction. After hydration, their electrical conductivities were studied at high pressure and high temperature to have a better understanding of the nature of proton transport in those materials.

Two batches of BZY10 were prepared, correspondingly by solid-state reaction (SS) ${ }^{9,10,15,16}$ and sol-gel (SG) synthesis. SG material was prepared starting from barium acetate (99\%), zirconium (IV) 2,4-pentanedionate (99\%) and yttrium (III) 2-ethylhexanoate $(99.8 \%)$; concentrated glacial acetic acid ( $>99 \%)$ being used as chelating agent and solvent. The reactants were dissolved in stoichiometric ratios in acetic acid at $353 \mathrm{~K}$ and the resulting clear solution was stirred for another $3 \mathrm{~h}$, followed by slow evaporation of the remaining acetic acid on a hot plate. The resultant dark brown syrup remained precipitate-free, leading to very fine powder by heating to $627 \mathrm{~K}$ and then to $1573 \mathrm{~K}$ to ensure the formation 


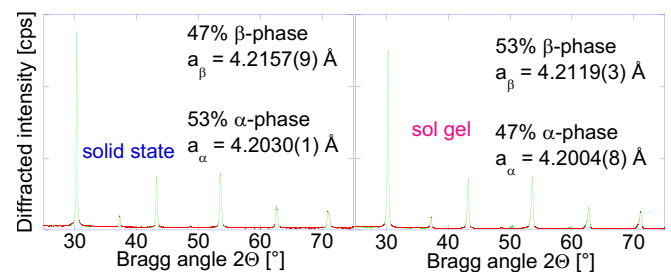

FIG. 1. (Color online) X-ray powder diffractograms of BZY10 prepared by SS and SG route.

of BZY10. Pellets of material were pressed and fired at 1773 $\mathrm{K}$ for $12 \mathrm{~h}$ for densification, and ground into powder for crystal structure and conductivity measurements.

It has been reported that, depending on synthesis parameters, two isostructural cubic $P m-3 m$ phases may coexist in BZY10: a so-called well conducting $\beta$-phase with a larger lattice constant $(a)$, and an $\alpha$-phase with a smaller lattice constant, as previously revealed using high resolution neutron diffraction data on samples prepared in similar conditions. ${ }^{17}$ This is confirmed by the Rietveld refinement of the x-ray diffractograms (see Fig. 1), showing that SS and SG samples are each comprised by two cubic structures with $P m-3 m$ space group, as evidenced by the clear splitting of the peaks: $47 \%$ with $a_{\mathrm{SS}}=4.2157(9) \AA$ and $53 \%$ with $a_{\mathrm{SS}}$ $=4.2030(1) \AA$ for SS, and 53\% with $a_{\mathrm{SG}}=4.2119(3) \AA$ and $47 \%$ with $a_{\mathrm{SG}}=4.2004(8) \AA$ for the SG sample. Line width analysis of the Bragg reflections by the Scherrer equation showed the average crystallite size for SS derived samples was $60-100 \mathrm{~nm}$, and $30-40 \mathrm{~nm}$ for the SG derived sample.

The samples were hydrated, also referred to as protonation, like described previously, ${ }^{3}$ i.e., the samples were heated to an optimized loading temperature of $723 \mathrm{~K}$ in $\mathrm{H}_{2} \mathrm{O} / \mathrm{N}_{2}$ flow for $24 \mathrm{~h}$. Electrical impedance spectra were obtained by Solartron 1260 Phase-Gain-Analyzer. A piston-cylinder apparatus was applied on protonated BZY10 powders, $1 \mathrm{GPa}$ $<\mathrm{p}<2 \mathrm{GPa}$ and $300 \mathrm{~K}<\mathrm{T}<680 \mathrm{~K}^{18}$ The analysis of impedance data follows our previous works. ${ }^{9,10,15,16}$

The temperature and pressure dependence of the conductivities for BZY10 prepared by SS and SG routes are shown respectively in Figs. 2(a) and 2(b). Particularly the low temperatures show that lower pressures provide a higher conductivity than the higher pressures, suggesting that "protons need space" in order to be mobile. This effect seems more pronounced for the low temperatures, where proton jump rotation is known to be the dominant mode, ${ }^{6}$ than for the high temperatures, where the effective jump diffusion sets on.

The $E_{a}$ for bulk and grain boundary conductivities, shown in Fig. 3, was determined by Arrhenius plot of the
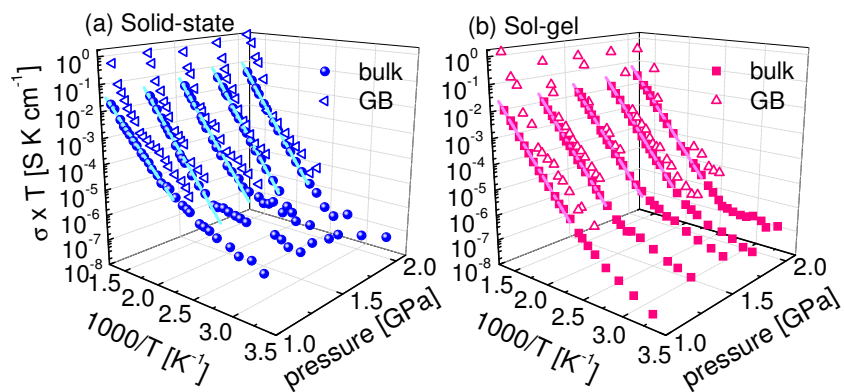

FIG. 2. (Color online) Conductivity of BZY10 at high pressure and high temperature. Bulk and grain boundary conductivity for SS derived samples (a); and for SG derived samples (b).

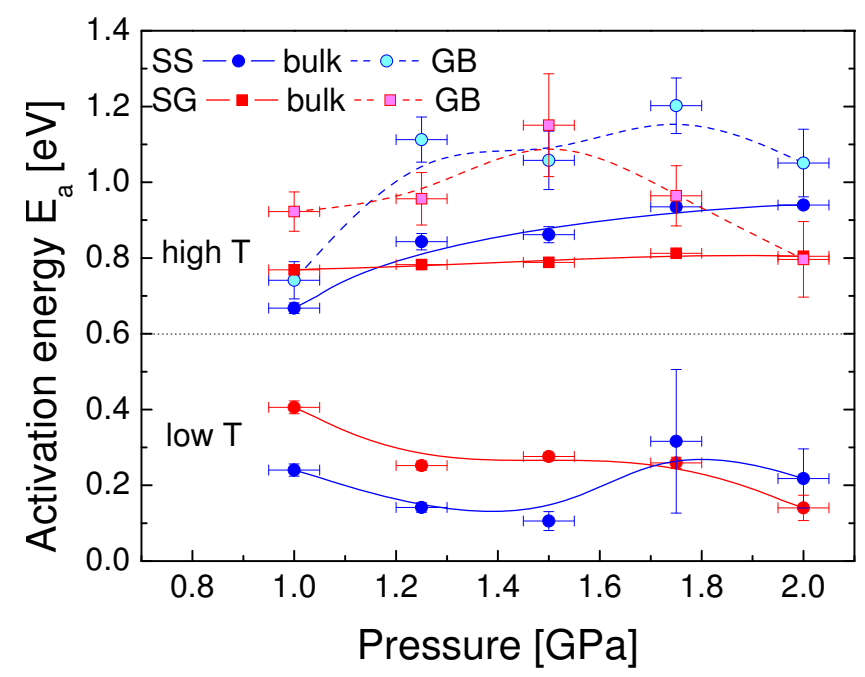

FIG. 3. (Color online) Activation energy of bulk and grain boundary conductivity for SS and SG synthesized samples at various pressures.

conductivity data in Fig. 2. At low $\mathrm{T}<420 \mathrm{~K}$, overall smaller $E_{a}$ were found; whereas at high temperatures the increase in $E_{a}$ indicated that the protons need to overcome an energy barrier to be conductive. This is in line with the previous interpretation of quasi-elastic neutron scattering (QENS) data, where in Arrhenius representation for low temperatures a flat range of proton diffusivity is found which is hardly to quantify with finite $E_{a}$ because it is in the resolution limit of the experiment. This behavior was assigned to the protons trapped in a localized state. ${ }^{6,19}$ For higher temperatures, the $E_{a}$ from QENS and impedance spectroscopy were found to be in a comparable range. ${ }^{6,719}$ The first observation is that the grain boundary $E_{a}$ displayed in Fig. 3 are for the probed pressure range systematically larger than those of the bulk conductivities. For the SS derived sample, bulk $E_{a}$ at $420 \mathrm{~K}<\mathrm{T}<680 \mathrm{~K}$ is $0.67 \mathrm{eV}$ for $1 \mathrm{GPa}$ and $0.94 \mathrm{eV}$ for $2 \mathrm{GPa}$, and $0.77-0.80 \mathrm{eV}$ for the SG derived sample, constituting a relative increase in $E_{a}$ by $40 \%$ and $5 \%$, respectively. The difference in $E_{a}$ between SS and SG prepared samples can be explained by their different proton concentration. The estimated water uptake of the SG sample from thermogravimetry data collected under wet $5 \% \mathrm{H}_{2}$ / Ar atmosphere from 300 to $1200 \mathrm{~K}$ is $0.02 \mathrm{~mol} \%,{ }^{20}$ thus we can assume it contains very few protons; whereas the proton concentration of the SS prepared sample equals $3 \mathrm{~mol} \%$ or more. ${ }^{10}$ Furthermore, no weight loss was found from TGA in dry air in the protonated SS samples up to $723 \mathrm{~K}$, which is above the maximum temperature applied during the impedance measurement.

In a recent study ${ }^{10}$ it was found that BZY10 of the nominal identical stoichiometry had $E_{a}$ ranging from 0.45 to 0.80 $\mathrm{eV}$, depending on how they were synthesized. They had the same crystallographic phase symmetry, but their lattice constants differed, which we interpret as a form of chemical pressure. Interestingly, the study showed a clear linear relation between the bulk $E_{a}$ and lattice constant. This striking correlation implies that the crystal lattice volume constitutes a very important parameter for bulk proton conductivity.

A necessary method to compare the transport properties of all aforementioned samples with respect to the pressure is to base them on their lattice constant. Since we do not have the necessary data for the lattice parameter of BZY10 as a function of pressure, we apply literature data for $\mathrm{BaZrO}_{3}$ by 


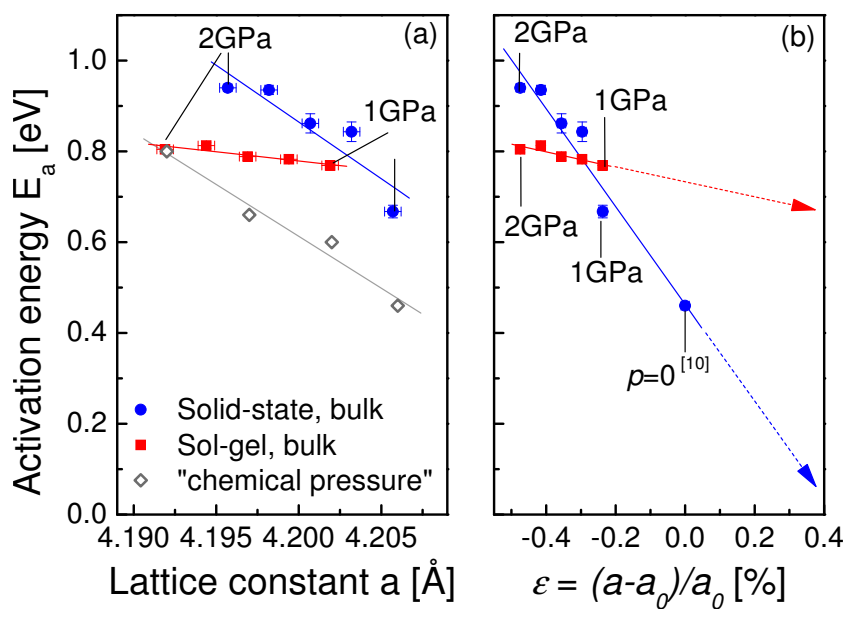

FIG. 4. (Color online) Variation in activation energy of bulk conductivity on the lattice parameter, under mechanical and "chemical" pressure (a); and on the strain parameter $\varepsilon$ (b). The data point at ambient pressure was taken from Ref. 10.

Kurosaki $^{21}$ et al. Combining the equilibrium lattice parameter of our SS and SG BZY10 samples from the $\beta$-phase of a much higher proton conductivity and their compressibility data $^{21}$ for $\mathrm{BaZrO}_{3}$, we translated pressure into lattice spacing. We assume here that the same pressure-dependent lattice parameter expansion applies for BZY10 at elevated temperature, thus it is possible to correlate the conductivity properties with the material lattice constant, as displayed in Fig. 4(a). At $1 \mathrm{GPa}$ pressure, the $\mathrm{SG}$ sample has an $E_{a}$ of $0.78 \mathrm{eV}$ and a lattice parameter of $4.1925 \AA$. The $E_{a}$ increases with increasing pressure to $0.80 \mathrm{eV}$ at $2 \mathrm{GPa}$ and $4.1825 \AA$. For the SS sample, the $E_{a}$ extends over a larger range and also with a steeper slope. This representation allows us to compare also with the samples obtained by different synthesis and temperatures. ${ }^{10}$

The open symbols in Fig. 4(a) denote the $E_{a}$ of the "chemical pressure" samples from ref. 10, which range from $0.45 \mathrm{eV}$ for $4.206 \AA$ to $0.8 \mathrm{eV}$ at $4.192 \AA$ with the slope. Similar to that from our SS samples. For all three classes of samples, it is evident that increasing lattice constant goes along with decreasing bulk $E_{a}$. Figure 4(a) shows now the general trend that the lowest $E_{a}$ goes along with the largest lattice constant realized by the lowest pressure. Applying hydrostatic pressure has the same effect like tuning the "chemical pressure" induced by different synthesis routes and sintering temperatures. ${ }^{10}$ It is confirmed that the $E_{a}$ decrease linearly with the increase in lattice constant. For the SG sample, the extent of lattice constant dependency is, however, not as large as found in ref. 10 .

The changes in $E_{a}$ in the grain boundary, both for SS and SG derived samples, are much more significant than those of bulk $E_{a}$ revealing that grain boundary tailoring is an efficient way to enhance proton conductivity. It appears like there is a maximum value for the grain boundary $E_{a} p=1.5 \mathrm{GPa}$ or $1.75 \mathrm{GPa}$. At $2 \mathrm{GPa}, E_{a}$ decreased to smaller values. This observation can be rationalized by a high pressure effect on the structure of grain boundary regions. For the SG derived samples, which have smaller crystallite size, this effect is more significant $\left(\sim 32 \%\right.$ decrease in $\left.E_{a}\right)$ than in the SS derived sample with larger crystallite size $(\sim 13 \%)$. Under high pressure, proton conductivity may be favored in the vicinity of grain boundaries. However, the error bar of the grain boundary $E_{a}$ is one order of magnitude higher than that from the bulk. Therefore, more temperature and pressure data are necessary for a better supported conclusion.

Our technological interest is of course not increasing the $E_{a}$ by compressing, but the opposite- expanding the lattice and thus potentially lowering $E_{a}$. Considering the variation in the bulk $E_{a}$ as a function of the reduced lattice parameter ratio $a / a_{0}$ or, like exercised in Fig. 4(b), on the strain parameter $\varepsilon=\left(a-a_{0}\right) / a_{0}$, it is suggested that proton conductors prepared under tensile strain, i.e., $\varepsilon>0$, could have an even lower $E_{a}$ and improved proton conductivity. Such situation could be realized in epitaxial strained films, for example, which also bears the possibility of having less grain boundaries that limit the proton conductivity. Stabilization of metastable proton conducting phases with tensile strain may, therefore, be a very rewarding research direction to be pursued further. It would be interesting to learn to what extent enhanced proton conductivity would be observed in expanded lattices and which additional material parameters would drive or impede this process.

Funding by E.U. MIRG Grant No. CT-2006-042095, Swiss NSF Grant No. 200021-124812, Swiss Federal Office of Energy under Project No. 100411, and Empa Director's Fund sixth R\&D Series. We are grateful to Joël Mesot (Paul Scherrer Institut) for helpful advice.

${ }^{1}$ K. D. Kreuer, Annu. Rev. Mater. Res. 33, 333 (2003)

${ }^{2}$ T. Norby, M. Widerøe, R. Glöckner, and Y. Larring, Dalton Trans. 19, 3012 (2004).

${ }^{3}$ A. Braun, A. Ovalle, V. Pomjakushin, A. Cervellino, S. Erat, W. C. Stolte, and T. Graule, Appl. Phys. Lett. 95, 224103 (2009).

${ }^{4}$ M. S. Islam, R. A. Davies, and J. D. Gale, Chem. Commun. (Cambridge) 2001, 661 .

${ }^{5}$ M. E. Björketun, P. G. Sundell, and G. Wahnström, Phys. Rev. B 76, 054307 (2007).

${ }^{6}$ T. Matzke, U. Stimming, C. Karmonik, M. Soetratmo, R. Hempelmann, and F. Guthoff, Solid State Ionics 86-88, 621 (1996).

${ }^{7}$ R. Hempelmann, C. Karmonik, T. Matzke, M. Cappadonia, U. Stimming, T. Springer, and M. A. Adams, Solid State Ionics 77, 152 (1995).

${ }^{8}$ T. Scherban, W. K. Lee, and A. S. Nowick, Solid State Ionics 28-30, 585 (1988).

${ }^{9}$ S. B. C. Duval, P. Holtappels, U. Stimming, and T. Graule, Solid State Ionics 179, 1112 (2008).

${ }^{10}$ S. B. C. Duval, Dissertation, Techn. Univ. München, 2008.

${ }^{11}$ A. Ashok, N. Kochetova, T. Norby, and A. Olsen, Solid State Ionics 179, 1858 (2008)

${ }^{12}$ J. Wu, Dissertation, Caltech, 2004.

${ }^{13}$ B. Merinov, C. O. Dorso, W. A. Goddard, J. Wu, and S. Haile, Report No. 833847, 2003 (unpublished).

${ }^{14}$ R. Hinrichs, G. Tomandl, and J. A. H. da Jornada, Solid State Ionics 77, 257 (1995).

${ }^{15}$ S. B. C. Duval, P. Holtappels, U. F. Vogt, E. Pomjakushina, K. Conder, U. Stimming, and T. Graule, Solid State Ionics 178, 1437 (2007).

${ }^{16}$ A. Braun, S. Duval, P. Ried, J. Embs, F. Juranyi, T. Strässle, U. Stimming, R. Hempelmann, P. Holtappels, and T. Graule, J. Appl. Electrochem. 39, 471 (2009).

${ }^{17}$ A. Azad, C. Savaniu, S. Tao, S. Duval, P. Holtappels, R. M. Ibberson, and J. T. S. Irvine, J. Mater. Chem. 18, 3414 (2008).

${ }^{18}$ N. Bagdassarov, H. C. Freiheit, and A. Putnis, Solid State Ionics 143, 285 (2001).

${ }^{19}$ R. Hempelmann, Physica B 226, 72 (1996).

${ }^{20}$ C. D. Savaniu, J. Canales-Vazquez, and J. T. S. Irvine, J. Mater. Chem. 15, 598 (2005).

${ }^{21}$ K. Kurosaki, J. Adachi, T. Maekawa, and S. Yamanaka, J. Alloys Compd. 407, 49 (2006). 